\title{
Temporal patterns of fire sequences observed in Canton of Ticino (southern Switzerland)
}

\author{
L. Telesca ${ }^{1}$, M. Kanevski ${ }^{2}$, M. Tonini ${ }^{2}$, G. B. Pezzatti ${ }^{3}$, and M. Conedera ${ }^{3}$ \\ ${ }^{1}$ Istituto di Metodologie per l'Analisi Ambientale, CNR, C. da S. Loja, 85050 Tito (PZ), Italy \\ ${ }^{2}$ Institut of Geomatics and Analysis of Risk, University of Lausanne, 1015 Lausanne, Switzerland \\ ${ }^{3}$ WSL Swiss Federal Research Institute, Insubric Ecosystems Research Group, via Belsoggiorno 22, \\ 6500 Bellinzona, Switzerland
}

Received: 2 September 2009 - Revised: 21 December 2009 - Accepted: 6 January 2010 - Published: 13 April 2010

\begin{abstract}
Temporal dynamical analysis in fire sequences recorded from 1969 to 2008 in Canton Ticino (Switzerland) was carried out by using the Allan Factor statistics. The obtained results show the presence of daily periodicities, superimposed to two time-scaling regimes. The daily cycle vanishes for sequences of higher altitude fires, for which a single scaling behaviour is observed.
\end{abstract}

\section{Introduction}

Fires are spatiotemporal events and their studies should be considered as complex spatiotemporal patterns. If the spatial aspect is not considered directly, fires can be represented as events occurring at random locations in time, thus, they can be modelled by a stochastic point process. Such studies can be important to real decision-making processes dealing with the distribution of fire prevention, pre-suppression and fighting efforts.

The self-organised criticality (SOC) is a good theoretical base for understanding the dynamics of fires (Clar et al., 1999). Fires are phenomena affecting a system (community, landscape unit, ecosystem, etc.) that can be considered as an extended dynamical system operating at states of critical equilibrium: the only spatial or temporal scales are those deduced from the size of the system (Bak et al., 1988). The main features of SOC systems are spatial fractality, temporal fluctuations scaling as $1 / f^{\alpha}$ and power-law frequency distribution of sizes. In the context of studies concerning fires, much attention has been focused on the problem of the fre-

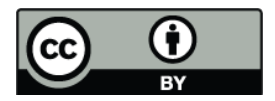

Correspondence to: $\mathrm{M}$. Tonini (marj.tonini@unil.ch) quency distribution of burned areas, leading to the more or less complex models fitting real observations (Malamud et al., 1998; Ricotta et al., 2001; Song et al., 2001; Reed and McKelvey, 2002). All these studies deal with one of the parameters that define a fire event, i.e. the size of burned area. However, the concept of self-organised criticality also implies that the time evolution of the system is time-scaling. Recently, several papers have focused on the temporal fluctuations of fire sequences, revealing the presence of scaling behaviour in the time dynamics of fire (Telesca et al., 2005; Corral et al., 2008) through the presence of power-law shape in the statistics describing the temporal distribution of fires.

In the present paper, the temporal fluctuations of forest fires observed in the Canton of Ticino (southern Switzerland) from 1969 to 2008 have been analysed using the Allan Factor method, which is well suited to detect scaling behaviours in point processes.

\section{Study area and dataset}

The Canton Ticino is located on the southern slope of the Alps and represents the southernmost of the 26 Swiss Cantons bordering on Italy (Fig. 1). It has a total area of $2812 \mathrm{~km}^{2} 2$, with 320000 inhabitants mostly located in the lowlands below $500 \mathrm{~m}$ a.s.l. Like the whole southern slope of the Alps, the area is characterised by a marked altitudinal gradient (from 200 to $3400 \mathrm{~m}$ a.s.l.) and quite a heterogeneous geology, dominated by siliceous rocks originated from the tectonics of the Alps. In the lowlands $(<1000$ m a.s.l.), the climate is influenced by the presence of consistent water masses (Lakes of Ceresio and Verbano) which results in particular climatic conditions (Insubric climate) characterised by dry and mild winters with some days ( 40 days a year on

Published by Copernicus Publications on behalf of the European Geosciences Union. 


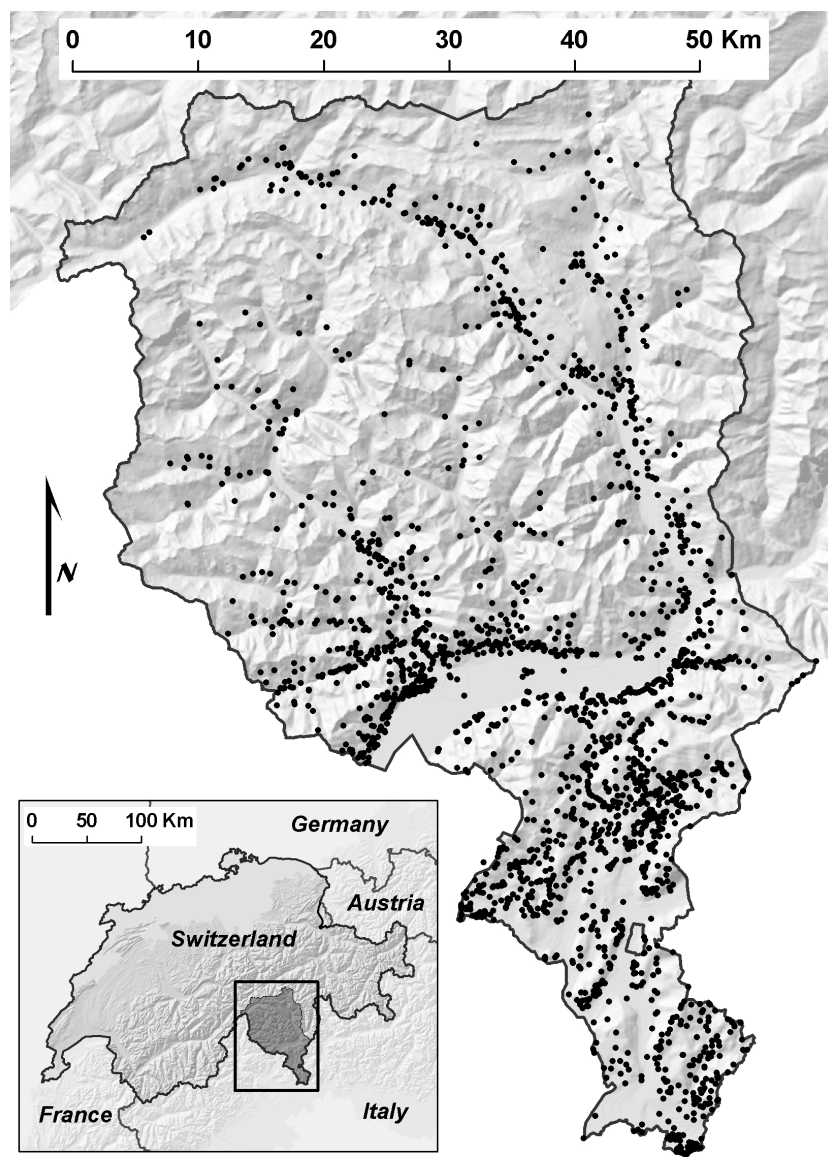

Fig. 1. The study area. Black dots represent the ignition points of the considered forest fires.

average) having strong gusts of a katabatic (descending) dry wind from the North (Foehn), which causes drops in the relative humidity to values as low as $20 \%$. The mean annual precipitation ranges from 1600 to $2100 \mathrm{~mm}$, and the mean annual temperature from 10 to $12^{\circ} \mathrm{C}$. In summer, long periods without rain or even drought may alternate with thunderstorms and short, heavy spells of precipitation. This results in a high quantity of summer rain $(800-1200 \mathrm{~mm}$ in the period June to September) that contrasts with the low level of summer precipitation in the Mediterranean climate. At a high elevation ( $>1500 \mathrm{~m}$ a.s.l.), the amount of annual precipitation is higher (2500-2600 $\mathrm{mm}$ ) and in winter characterised by snow. Mean annual temperature drop to $5^{\circ} \mathrm{C}$ or less (Spinedi and Isotta, 2004). Figure 2 reports representative climatic diagrams for the different elevation horizons studied.

Forest cover of the area is high (on average 50.5\%). The forest vegetation is dominated at low elevations (up to $1000 \mathrm{ma.s.1}$.) by the chestnut tree (Castanea sativa), which was first cultivated (and probably first introduced) in the area by the Romans (Conedera et al., 2004). Chestnut forests are anthropogenic monocultures occasionally interrupted by the presence of other broadleaved species such as
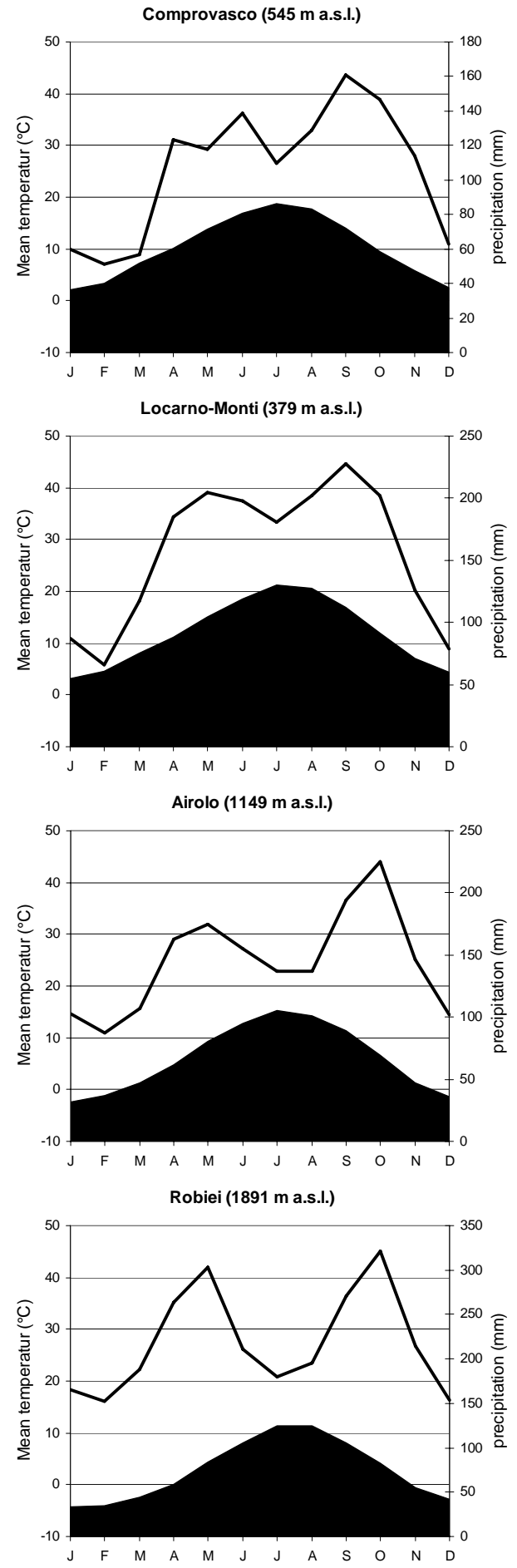

Fig. 2. Climatic diagrams referring to the period from 1970 to 2000 for representative meteorological stations of the study area (source: Spinedi and Isotta, 2004).

Tilia cordata, Quercus petraea, Q. pubescens, Alnus glutinosa, Prunus avium, Acer spp., or Fraxinus spp. At medium elevations (1000-1500 m a.s.1.), the forests mostly consist of pure stands of Fagus sylvatica. In this altitudinal horizon, 
the presence of Abies alba has been reduced to small patches on north-facing slopes in the central part of the study area. Scots pine (Pinus sylvestris) stands are confined to very particular sites on dry south-facing slopes. At high altitudes ( $>1500 \mathrm{~m}$ a.s.l.), coniferous forests dominates. The main species is the Norway spruce (Picea abies) followed by pure larch stands (Larix decidua) at very high elevation (>2000 m a.s.l.) and on alpine pasture land. Swiss stone pine (P. cembrae) dominates in small patches on the most continental areas of the upper regions (Ceschi, 2006). As a result, the different forest vegetation types are distributed following a marked altitudinal gradient with different vegetation belts according to the elevation (see Pezzatti et al., 2009 for further details).

Basic information on forest fires in Ticino (such as the date, time, and cause of ignition, fire duration, burned area, fire type, and forest type) has been collected by the Forest Service since 1900. Starting in 1969, georeferenced information on ignition point and burnt area have also been collected. All existing fire data are now organized and stored in a relational database (Pezzatti et al., 2005). For the present study, all the recorded fires from January 1969 to August 2008 are considered.

The entire dataset has been analysed by means of geographical information systems (GIS), namely ArcGis (ESRI). This has permitted us to select and extract the point of origin of fires in Ticino during the period of 1969-2008 (Fig. 1). An algorithm implemented in the Spatial Analyst extension has permitted us to calculate the altitude of each ignition point by extracting the cell values of a raster graphic image based on a set of points. The digital elevation model (DEM) with a resolution of $25 \mathrm{~m}$, has been used for this purpose. The DEM25 has been furnished by the Swiss Federal Office of Topography (Géodonnées@ Swisstopo (DV084371)).

Current monthly distribution of fires, as shown in Fig. 3, highlights the existence of two peaks. The major peak appears in March-April during the vegetation rest (DecemberApril), and the second peak in summer, July-August. When considering fires at elevations higher than $1000 \mathrm{~m}$ or $1500 \mathrm{~m}$, the number of fires is very small and only a slight summer peak is visible.

\section{Method}

There are many methods to study clustering of temporal data and point processes/events (Lowen and Teich, 2005). One of the well-known methods to investigate the temporal properties of a time series is the power spectral density (PSD) $S(f)$, obtained by means of a Fourier Transform (FT) of the series. The PSD gives information on how the power of the series is concentrated at various frequency bands. This information allows identifying periodic, multi-periodic or non-periodic frequency patterns. Usually the power spectrum plotted in

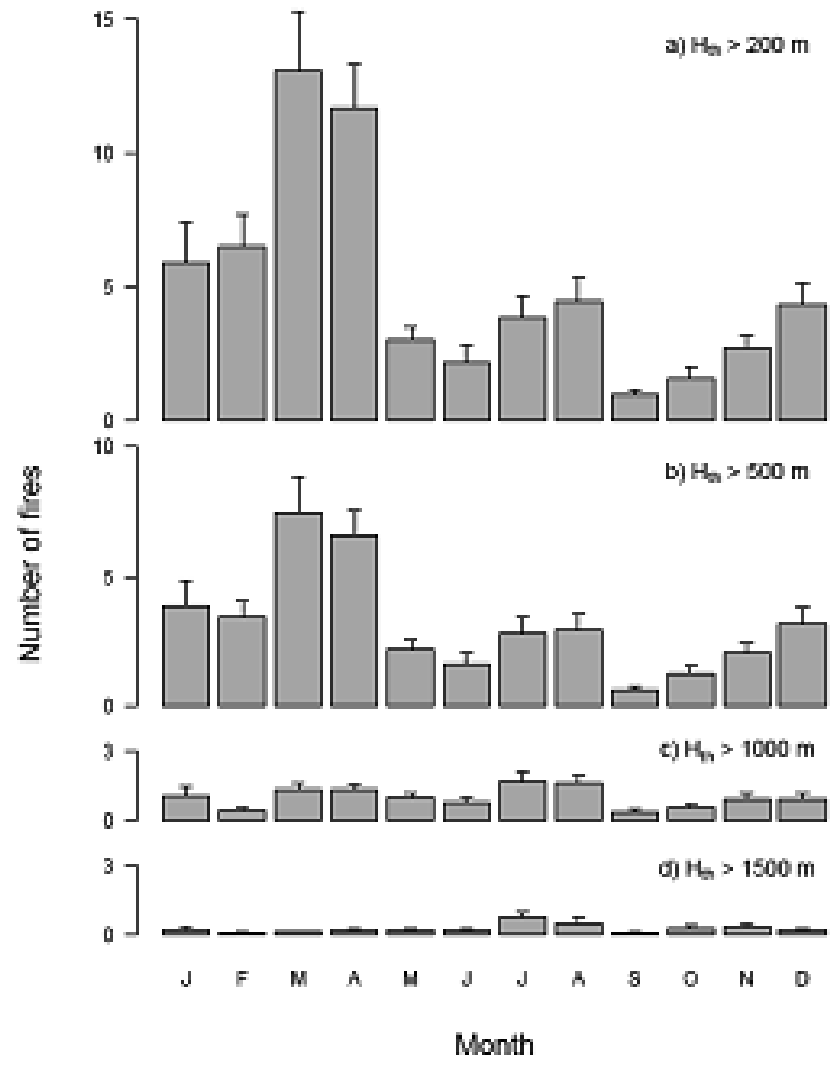

Fig. 3. Monthly fire distributions 1969-2008 according to the different elevation thresholds. The bars correspond to the standard errors.

$\log -\log$ scales is used to analyse broadband behaviour. The power-law (or fractal) dependence (linear on a log-log plot) of the PSD is given by $S(f) \sim f^{-\alpha}$ and is a hallmark of the presence of time-scaling in the data. The properties of the series can be further classified in terms of the numerical value of the spectral (or fractal) exponent: $\alpha=0$ features white noise time series, characterised by the absence of time correlations; while $\alpha \neq 0$ is typical of pink noise time series, characterised by the presence of time correlations.

A fire sequence is a temporal point process, given by a set of events occurring at random locations in time (Cox and Isham, 1980), and the simple application of the FT is not possible. The fire data can be represented by a finite sum of Dirac's delta functions centred on the occurrence times $t_{i}$, with amplitude $A_{i}$ proportional to the burned area:

$y(t)=\sum_{i=1}^{N} A_{i} \delta\left(t-t_{i}\right)$

where $N$ represents the number of events recorded.

Then dividing the time axis into equally spaced contiguous counting windows of duration $\tau$, which is called a timescale, a sequence of counts $\left\{N_{k}(\tau)\right\}$ is produced, with 


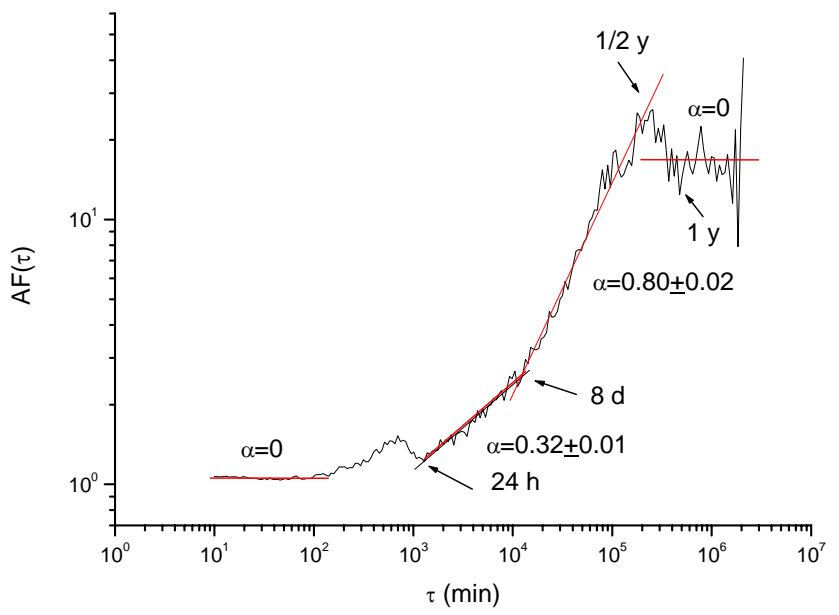

Fig. 4. AF for the whole data.

$N_{k}(\tau)$ denoting the number of fire events in the $k$-th window (Lowen and Teich, 1999):

$N_{k}(\tau)=\int_{t_{k}}^{t_{k+\tau}} \sum_{j=1}^{n} \delta\left(t-t_{j}\right) d t$,

The following quantity, the Allan Factor (AF), can be defined as

$\operatorname{AF}(\tau)=\frac{<\left(N_{k+1}(\tau)-N_{k}(\tau)\right)^{2}>}{2<N_{k}(\tau)>}$,

which is related to the variability of successive counts (Thurner et al., 1997); the symbol $<\ldots>$ indicates the average value. The AF has been largely used to investigate the time dynamics of earthquakes (Telesca et al., 2000, 2004).

If the point process is time-correlated, then the $\mathrm{AF}$ varies with the timescale $\tau$ with a power-law form:

$\operatorname{AF}(\tau)=1+\left(\frac{\tau}{\tau_{1}}\right)^{\alpha}$,

over a certain range of timescales $\tau$; and the exponent $\alpha$ quantifies the strength of time-correlation; $\tau_{1}$ is the fractal onset time and marks the lower limit for significant scaling behaviour in the AF, so that for $\tau \ll \tau_{1}$ the time-scaling property becomes negligible at these time scales (Lowen and Teich, 1996, 2005). AF assumes values near unity for Poisson processes (Thurner et al., 1997). Therefore, if $\alpha=0$ the point process is Poissonian, which means that the series is memoryless and constituted by independent events; while if $\alpha>0$ the process is characterised by time-scaling behaviour, which means that the series is time-correlated.

\section{Results}

During the observation period, 2401 forest fires have been recorded. In particular the time span from 3 January 1969

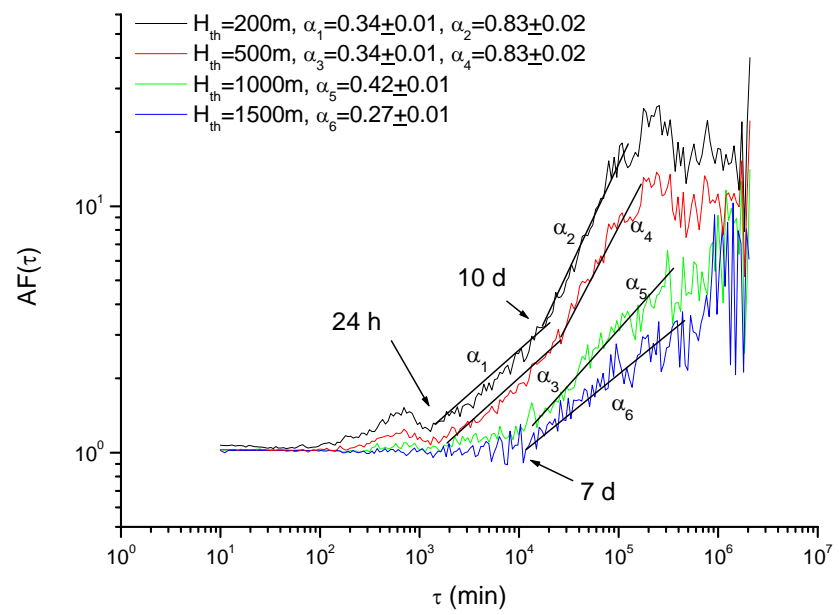

Fig. 5. AF for different elevation thresholds.

to 8 August 2008. Figures 4 and 5 shows the relations $\operatorname{AF}(\tau) \sim \tau$ in log-log scales for the sequences. Equation (3) has been calculated for timescales $\tau$ ranging between $10 \mathrm{~min}$ and $\mathrm{T} / 10$, where $\mathrm{T}$ is the total observation period of the fire catalogue. The analysis of the AF curve allows getting insight into several features characterising the analysed fire sequence:

1. The fire activity of Ticino is not Poissonian, because the AF curve is not flat for all the timescales $\tau$. This result indicates the presence of time-clustering behaviour in the fire dynamics, which implies the presence of timecorrelation structures in the time distribution of the fire events. Moreover, the presence of crossovers (separating different regions with different slopes) indicates the co-existence of different dynamics in the same fire process.

2. The fire sequence shows different time regimes. a) At a small timescale, the behaviour is Poissonian, because the $\mathrm{AF}$ is almost constant with value around the unity. This is typical for point processes, when the timescale investigation is very small. b) Two scaling regions are identified in the curve and the scaling exponents are 0.32 in the small timescale, $1-8$ days, and 0.8 in the large timescale, 8 days $-1 / 2$ year. This phenomenon indicates the superimposition of different dynamics and the crossover at about 8 days could be related to anthropic activities (Wang et al., 2010). c) The large timescales, over $1 / 2$ year, seem to show a purely random behaviour, since the AF curve is approximately flat, although this effect can also be produced by the lower resolution corresponding to a larger timescale. However, no clustering effect seems to be detected at larger timescales. 
3. The sequence of fires show the presence of the daily and annual periodicities, indicated by the two drops in the AF curve.

4. Increasing the altitude of the fires, the two-fold scaling behaviour and the daily cycle tend to vanish, with a decrease in the estimate of the scaling exponent.

\section{Discussion and conclusions}

The AF applied to the fire sequence recorded in Canton Ticino has revealed the presence of several scaling regimes along with daily and annual periodicities.

The presence of different scaling regimes suggests the existence of different dynamical mechanisms governing the fire sequence, with different strengths of the power-law fluctuations, indicated by the value of the scaling exponent. The different mechanisms could be due to mixed natural and anthropogenic causes. The periodicity at about 1 day can be placed in relation to both the diurnal meteorological cycle and the diurnal anthropogenic behaviour. Pippen (1999) and Plucinski (1999) found a diurnal variation in the state of the deadfuel moisture content of open woodland fuel, in response to the normal diurnal weather changes, such as the rise and fall of temperature and solar radiation. In Ticino, Conedera et al. (1996) found a one hour shift in the starting time of human-caused fires since 1981 as a consequence of the introduction of the legal summer time. The annual frequency represents the typical seasonal variability, due to cumulative effects of weather, climate as well as normal biophysiological lifecycles. The vegetation, in particular the litter and the herb layer in the forest stand understory, has a seasonal growth cycle. As expected, the state of litter and herb layer has an important influence on the ignition and spreading of fires. Both periodicities tend to vanish with the increase of the altitude of the fires, which could suggest the prevailing of different meteo-climatic effects, probably characterised by fluctuations with characteristic timescales longer than the annual periodicity. However, the lower anthropogenic activity and different vegetation cover at higher altitudes should also be taken into account. In fact, the single-scaling behaviour at high altitudes could be due to the predominance of natural-caused (lightning) fires. At these altitudes, the scaling exponent ranges around 0.3 , which is in good agreement with the scaling exponent calculated for lightning sequences at timescales consistent with those characterising these fires (Telesca et al., 2005).

The obtained results show the relevance of applying temporal fractal analyses to fire distributions in order to better understand the underlying dynamical mechanisms.

The ongoing and future research deal with the comparison of the Alan factor with other measures of temporal clustering (Morisita index, fractal clustering, scan statistics, etc.) as well as with the complete analysis of spatiotemporal patterns of the studied phenomena.
Acknowledgements. One of the authors, L. Telesca, acknowledges the support received in 2009 by CNR and FNS (Project N. IZAIZO12777) in the framework of the scientific bilateral cooperation CNR/FNS. The project was also partly supported by the Swiss FNS project “GeoKernels 2" (200020-121835).

Edited by: R. Lasaponara

Reviewed by: M. Lovallo and two other anonymous referees

\section{References}

Bak, P., Tang, C., and Wiesenfeld, K.: Self-organized criticality, Phys. Rev. A, 38, 364-374, 1998.

Clar, S., Drossel, B., Schenk K., and Schwabl, F.: Self-organized criticality in forest-fire models, Physica A, 266, 153-159, 1999.

Ceschi, I.: Il bosco nel Canton Ticino. Armando Dadò Editore: Locarno, 407 pp., 2006 (in Italian).

Corral, A., Telesca, L., and Lasaponara, R.: Scaling and correlations in the dynamics of forest-fire occurrences, Phys. Rev. E, 77, 016101, 2008

Conedera, M., Marcozzi, M., Jud, B., Mandallaz, D., Chatelain, F., Frank, C., Kienast, F., Ambrosetti, P., and Corti, G.: Incendi boschivi al Sud delle Alpi: passato, presente e possibili sviluppi futuri. In: Rapporto di lavoro del Programma Nazionale di Ricerca "Mutamenti climatici e catastrofi naturali" PNR 3. Zürich, vdf Hochschulverlag, 1996.

Conedera, M., Krebs, P., Tinner, W., Pradella, M., and Torrioni, D.: The cultivation of Castanea sativa (Mill.) in Europe: from its origin to its diffusion on a continental scale, Veg. Hist. Archaeobot., 13, 161-179, 2004.

Cox, D. R. and Isham, V.: Point Processes, London: Chapman and Hall, 1980.

Lowen, S. B., and Teich, M. C.: Estimation and Simulation of Fractal Stochastic Point Processes, Fractals, 3, 183-210, 1999.

Lowen, S. B. and Teich, M. C.: The Periodogram and Allan Variance Reveal Fractal Exponents Greater than Unity in AuditoryNerve Spike Trains, J. Acoust. Soc. Am., 99, 3585-3591, 1996.

Lowen, S. and Teich, M.: Fractal-Based Point Processes, WileyInterscience, New Jersey, 2005.

Malamud, B. D., Morein, G., and Turcotte, D. L.: Forest fires: An example of self-organised critical behaviour, Science, 281, 1840-1842, 1998

Pezzatti, G. B., Conedera, M., and Kaltenbrunner, A.: Die neue Waldbranddatenbank, Bündnerwald, 58, 37-39, 2005.

Pezzatti, G. B., Bajocco, S., Torrioni, D., and Conedera, M.: Selective burning of forest vegetation in Canton Ticino (southern Switzerland), Plant Biosyst., 143(3) 609-620, 2009.

Pippen, B. G.: Predicting fine fuel moisture in shrubby vegetation, Honours thesis, Australian National University, 1999.

Plucinski, M. P.: The investigation of factors governing ignition and development of fires in heatland vegetation, Ph.D. thesis, University of New South Wales, ADFA, 1999.

Reed, W. J. and McKelvey, K. S.: Power-law behaviour and parametric models for the size-distribution of forest fires, Ecol Model., 150, 239-254, 2002.

Ricotta, C., Arianoutsou, M., Diaz-Delgado, R., Duguy, B., Lloret, F., Maroudi, E., Mazzoleni, S., Moreno, J. M., Rambal, S., 
Vallejo, R., and Vazquez, A.: Self-Organized criticality of wildfires ecologically revisited, Ecol. Model., 141, 307-311, 2001.

Spinedi, F. and Isotta, F.: Il clima del Ticino negli ultimi 50 anni Dati statistiche e società, 4, 4-39, 2005.

Song, W., Weicheng, F., Bighong, W., and Jianjun, Z.: Selforganized criticality of forest fire in China, Ecol. Model., 145, 61-68, 2001.

Telesca, L., Cuomo, V., Lapenna, V., and Macchiato, M.: Analysis of time-scaling behaviour in the sequenze of the aftershocks of the Bovec (Slovenia) April 12, 1998 earthquake, Phys. Earth Planet. Int., 120, 315-326, 2000.

Telesca, L., Lapenna, V., Lovallo, M., and Macchiato, M.: Longrange time-correlation properties of seismic sequences, Chaos Solition Fract., 21, 387-393, 2004.
Telesca, L., Amatulli, G., Lasaponara, R., Lovallo, M., and Santulli, A.: Time-scaling propertiesin forest-fire sequences observed in Gargano are (southern Italy), Ecolog. Model., 185, 531-544, 2005.

Telesca, L., Bernardi, M., and Rovelli, C.: Intra-cluster and intercluster time correlations in lightning sequences, Physica A, 356, 655-661, 2005.

Thurner, S., Lowen, S. B., Feurstein, M. C., Heneghan, C., Feichtinger, H. G., and Teich, M. C.: Synthesis, and Estimation of Fractal-Rate Stochastic Point Processes, Fractals, 5, 565-595, 1997.

Wang, J., Song, W., Zheng, H., Telesca, L.:Temporal scaling behavior of human-caused fires and their connection to relative humidity of the atmosphere, Ecol. Model., 221, 85-89, 2010. 